\title{
ALL SINGULARITIES OF THE 9-DOF DLR MEDICAL ROBOT SETUP FOR MINIMALLY INVASIVE APPLICATIONS
}

\author{
Rainer Konietschke ${ }^{1}$, Gerd Hirzinger \\ German Aerospace Center, Institute of Robotics and Mechatronics \\ P.O. Box 1116, D-82230 Weßling, Germany \\ $1_{\text {Rainer.Konietschke@dlr.de }}$
}

Yuling Yan

University of Hawaii at Manoa, Mechanical Engineering

2540 Dole Street - Holmes Hall 302, Honolulu HI 96822, USA

\begin{abstract}
This paper shows that it is possible to determine analytically all singular configurations of the 9-DoF DLR medical robot setup for minimally invasive applications. It is shown that the problem can be devided into the determination of the singularities of the general 7-DoF DLR medical arm and of the 2-DoF surgical instrument, used in a minimally invasive application. The formula of Cauchy-Binet is used to calculate the singularities of the redundant medical arm, and an interpretation of this formula for any serial redundant robot design is given.
\end{abstract}

Keywords: medical robotics, singularities, manipulability, robotic assistance, minimally invasive surgery, optimization, robot design

\section{Introduction}

In robotically assisted minimally invasive applications, a surgical robot is used to access the operating field inside the human body through small incisions with thin cylindrical instruments. The design of such robotic devices for medical applications is liable to exceptionally high requirements in terms of safety and reliability. A thorough analysis of the robot's kinematic structure is important to ensure complete reachability as well as the absence of any singular configuration inside the desired workspace. The desired workspace is usually defined by the operator during a planning step, and serves to determine the optimal robot setup (Adhami, 2002; Konietschke et al., 2004). The robot setup comprises the position and orientation of the robot base and the position of the entry point into the human body as well as any adjustable DH parameter (as for example adjustable instrument lengths). 
The determination of the singular configurations of a robot is especially important in the case of teleoperation, where the exact path is not known in advance. Though singular configurations can be detected by monitoring certain manipulability measures as eg. in Yoshikawa, 1990; Konietschke et al., 2004, these measures are to the author's knowledge insufficient to signal vicinity to singular configurations. Since the behaviour of robots near singularities is in most cases not very intuititive for the operator, it is highly desirable to restrict the workspace admissible to the operator to a space that does not contain any singularities or to control the robot in a way that singular configurations are avoided. This is facilitated if an analytic description of all singularities of the robot design is known, since the use of computationally cheap strategies for singularity avoidance in analogy with well known strategies for joint limit avoidance becomes possible.

In the next section, the kinematic structure of the considered robotic system is presented. The singularities of the DLR medical arm and the attached surgical instrument are given in the sections 3 and 4 . Section 5 gives a short conclusion.

\section{Kinematic structure}

The kinematic structure of the considered robot with the attached actuated instrument and the used coordinate frames are shown in Fig. 1. The medical robot itself has $7 \mathrm{DoF}\left(\phi_{1 \ldots 7}\right)$ and the attached instrument disposes of two additional DoF $\left(\phi_{8,9}\right)$. The kinematic chain of the robot itself is denoted thereafter as $K_{1}$, that of the actuated instrument as $K_{2}$.

In the following, the problem of determining the singular configurations of the robot kinematics is divided into two subproblems. This is possible due to the restrictions at the entry point (see section 4).

\section{The singular configurations of the DLR medical arm}

Written in the wrist frame $\{\mathbf{W}\}$, the geometric Jacobian $\mathbf{J}$ of the forward kinematics has the following form (Yoshikawa, 1990):

$$
\left(\begin{array}{c}
\mathbf{v}_{W} \\
\omega_{W}
\end{array}\right)=\mathbf{J} \dot{\phi}=\left(\begin{array}{cc}
\mathbf{J}_{11} & \mathbf{0} \\
\mathbf{J}_{21} & \mathbf{J}_{22}
\end{array}\right) \dot{\phi}, \quad \text { with } \quad\left(\begin{array}{c}
\mathbf{v}_{W} \\
\omega_{W}
\end{array}\right)
$$

the translational and rotational velocity of the wrist frame $\{\mathbf{W}\}$ and

$$
\mathbf{J}_{11}, \mathbf{J}_{21} \in \mathbb{R}^{3 \times 4}, \mathbf{J}_{22} \in \mathbb{R}^{3 \times 3} .
$$

A singular configuration occurs if the following determinant equals zero: 


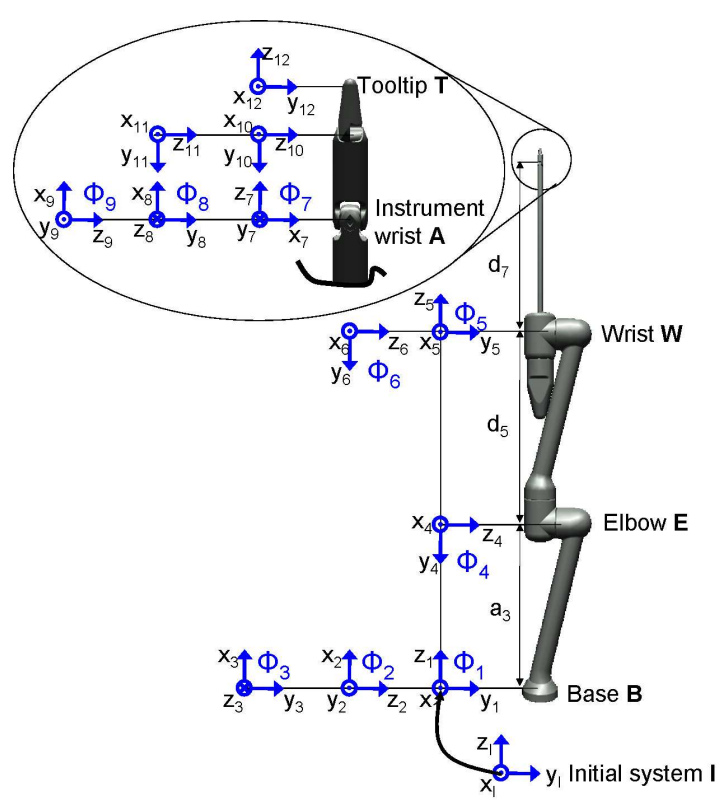

Figure 1. Kinematic description of the considered kinematic chains ( $K_{1}$ and $\left.K_{2}\right)$

$$
\left|\mathbf{J J}^{\mathbf{T}}\right|=0 .
$$

With the formula of Cauchy-Binet (see eg. Gantmacher, 1959), Eq. 3 can be transformed into a sum of squares of determinants:

$$
\left|\mathbf{J J}^{\mathbf{T}}\right|=\sum_{i=1}^{4}\left|\left(\begin{array}{cc}
\mathbf{J}_{11}^{i} & \mathbf{0} \\
\mathbf{J}_{21}^{i} & \mathbf{J}_{22}
\end{array}\right)\right|^{2}+\sum_{i=1}^{3}\left|\left(\begin{array}{cc}
\mathbf{J}_{11} & \mathbf{0} \\
\mathbf{J}_{21} & \mathbf{J}_{22}^{i}
\end{array}\right)\right|^{2},
$$

with $\mathbf{J}_{m n}^{i}$ the $i$-th submatrix (minor) obtained by suppressing column $i$ of the matrix $\mathbf{J}_{m n}$. The terms of the first sum have a lower block triangular form and can be combined to:

$$
\sum_{i}\left|\left(\begin{array}{cc}
\mathbf{J}_{11}^{i} & \mathbf{0} \\
\mathbf{J}_{21}^{i} & \mathbf{J}_{22}
\end{array}\right)\right|^{2}=\left|\mathbf{J}_{22}\right|^{2} \sum_{i}\left|\mathbf{J}_{11}^{i}\right|^{2}=\left|\mathbf{J}_{22}\right|^{2}\left|\mathbf{J}_{11} \mathbf{J}_{11}^{\mathbf{T}}\right| .
$$

In the last step, the formula of Cauchy-Binet is applied inversely. Since the sum in Eq. 4 consists of squared summands, all of them have to equal zero in a singular configuration. Simplifications are possible with consideration of the rank of the Jacobian. Due to the special structure of $\mathbf{J}$, a sufficient condition for a singular configuration is:

$$
\operatorname{rank}\left(\mathbf{J}_{11}\right)<3 \text {. }
$$


For the remaining singular configurations, a necessary condition is:

$$
\operatorname{rank}\left(\mathbf{J}_{22}\right)<3 .
$$

Thus, the second sum of Eq. 4 has to be evaluated only for joint angles that cause $\left|\mathbf{J}_{22}\right|$ to be zero. The following singularities $e_{i}$ can thus be determined, with $k \in \mathbb{N}$ :

$$
\begin{gathered}
e_{1}: \quad \phi_{4}=\pi k, \\
e_{2}: \quad \phi_{2}=\frac{\pi}{2}+\pi k \wedge \phi_{3}=\frac{\pi}{2}+\pi k, \\
e_{3}: \quad \phi_{2}=\frac{\pi}{2}+\pi k \wedge \phi_{4}= \pm \arccos \left(-\frac{a_{3}}{d_{5}}\right)+2 \pi k, \\
e_{4}: \quad \phi_{2}=\frac{\pi}{2}+\pi k \wedge \phi_{6}=\pi k, \text { and } \\
e_{5}: \quad \phi_{5}=\frac{\pi}{2}+\pi k \wedge \phi_{6}=\pi k .
\end{gathered}
$$

The singular configuration $e_{3}$ only appears if $\left\|a_{3}\right\| \leq\left\|d_{5}\right\|$. Details about the zero points of the relevant determinants are given in the appendix. The classical "wrist singularity" $\left(\phi_{6}=\pi k\right)$ that occurs in many 6-DoF kinematic chains (consider for example a kinematic chain $K_{1}^{\prime}$ obtained with joint $\phi_{3}$ held constant) does only appear in conjunction with additional conditions (Singularities $e_{4,5}$ ). To illustrate this, the pseudo inverse $\mathbf{J}_{a}^{+}$of the Jacobian $\mathbf{J}_{a}$ in the non singular configuration $\phi_{a}=(0,0,0, \pi / 2,0,0,0)^{\mathbf{T}}$ as shown left in figure 2 is considered, written in frame $\{\mathbf{I}\}$ :

$$
\mathbf{J}_{a}^{+}=\mathbf{J}_{a}^{\mathbf{T}}\left(\mathbf{J}_{a} \mathbf{J}_{a}^{\mathbf{T}}\right)^{-1}, \mathbf{J}_{a}^{+}=\left(\begin{array}{cccccc}
0 & 0 & 0 & 0 & 0 & 1 \\
\frac{1}{a_{3}} & 0 & 0 & 0 & 0 & 0 \\
0 & \frac{1}{a_{3}} & 0 & 0 & 0 & -\frac{d_{5}}{a_{3}} \\
-\frac{1}{a_{3}} & 0 & -\frac{1}{d_{5}} & 0 & 0 & 0 \\
0 & \frac{1}{2 a_{3}} & 0 & \frac{1}{2} & 0 & -\frac{d_{5}}{2 a_{3}} \\
0 & 0 & \frac{1}{d_{5}} & 0 & 1 & 0 \\
0 & \frac{1}{2 a_{3}} & 0 & \frac{1}{2} & 0 & -\frac{d_{5}}{2 a_{3}}
\end{array}\right) .
$$

With $d_{5} / a_{3} \approx 1$, all joint velocities remain small for arbitrary rotations of the tool tip. Particularly, pure rotation around an axis $b$ as shown left in Fig. 2 (perpendicular to the rotation axes of $\phi_{6}$ and $\phi_{7}$ and intersecting them), constituting the singular direction in case of a 

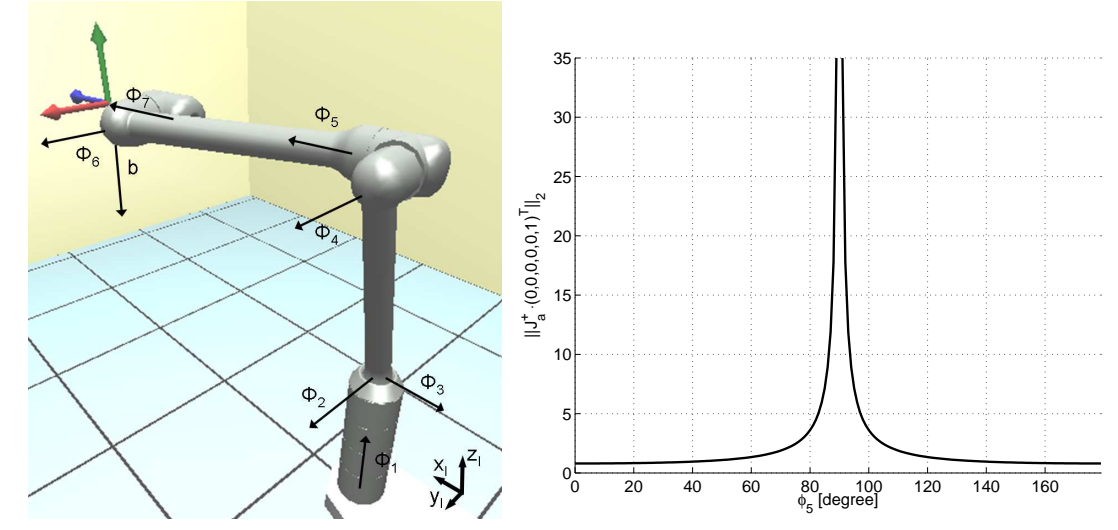

Figure 2. In case of the considered robot, pure rotations around the axis $b$ can still be performed even if $\phi_{6}=0$ (left). Only if also $\phi_{5}=\pi / 2+\pi k$, a singular configuration occurs, as can be seen on the right where the norm $\left\|\mathbf{J}_{a}^{+} \cdot(0,0,0,0,0,1)^{\mathbf{T}}\right\|_{2}$ is shown as a function of the joint angle $\phi_{5}$.

kinematic chain as $K_{1}^{\prime}$ in this configuration, leads to the following (reasonably small) joint velocities:

$$
\dot{\phi}=\mathbf{J}_{a}^{+} \cdot(0,0,0,0,0,1)^{\mathbf{T}}=\left(1,0,-\frac{d_{5}}{a_{3}}, 0,-\frac{d_{5}}{2 a_{3}}, 0,-\frac{d_{5}}{2 a_{3}}\right)^{\mathbf{T}} .
$$

On the right of Fig. 2 the norm $\left\|\mathbf{J}_{a}^{+} \cdot(0,0,0,0,0,1)^{\mathbf{T}}\right\|_{2}$ is shown as a function of the joint angle $\phi_{5}$ with all other angles remaining in configuration $\phi_{a}$.

\subsection{Generalisation to the case of a serial robot with $\mathrm{n}$-fold redundancy}

The singular configurations of a general, $n$-fold redundant robot can be calculated by considering the roots of the following determinant:

$$
\left|\mathbf{J J}^{\mathbf{T}}\right|=\sum_{i=1}^{\frac{(m+n) !}{2(m !)}}\left|\mathbf{J}_{i}\right|^{2}, \quad \mathbf{J} \in \mathbb{R}^{m \times(m+n)},
$$

with $\mathbf{J}_{i}$ representing all $\frac{(m+n) !}{2(m !)}$ (different) matrices obtainable by suppressing $n$ columns of the Jacobian $\mathbf{J}$. It can be seen from Eq. 15 that the singularities of a serial redundant structure with $m+n$ joints of which $n$ are redundant are identical with the intersection of the singularities of all those robotic structures obtained by fixing any possible set of $n$ joints 
of the redundant structure. It has to be noted however, that already for the case of a 2 -fold redundant robot with $8 \mathrm{DoF}, \frac{8 !}{2 \cdot 6 !}=28$ minors have to be considered, each of which being usually a rather complex function of the joint angles $\phi$.

\section{Singularities of the instrument in a minimally invasive application}

The kinematics in minimally invasive applications have the peculiarity of a fulcrum point where the surgical instrument enters into the human body. At that point, a constraint is imposed upon the system, resulting in a loss of two DoF. In order to regain full dexterity inside of the patient, an articulated instrument can be used, adding two DoF ( $\phi_{8}$ and $\phi_{9}$, see Fig 3) to obtain full $6 \mathrm{DoF}$ at the distal end of the instrument. To analyze the singular configurations introduced by the fulcrum point and the two extra DoF of the instrument, the following Jacobian matrix is considered:

$$
\left(\begin{array}{c}
\mathbf{v}_{9} \\
\omega_{9}
\end{array}\right)={ }_{9}^{6} \mathbf{J}_{v}\left(\begin{array}{c}
\dot{x}_{6} \\
\dot{y}_{6} \\
\dot{z}_{6} \\
\dot{\phi}_{7} \\
\dot{\phi}_{8} \\
\dot{\phi}_{9}
\end{array}\right),{ }_{9}^{6} \mathbf{J}_{v}=\left(\begin{array}{cccccc}
\frac{d_{7}^{\prime}-d_{7}}{d_{7}^{\prime}} & 0 & 0 & 0 & 0 & 0 \\
0 & 1 & 0 & 0 & 0 & 0 \\
0 & 0 & \frac{d_{7}^{\prime}-d_{7}}{d_{7}^{\prime}} & 0 & 0 & 0 \\
0 & 0 & \frac{1}{d_{7}^{\prime}} & 0 & -c_{7} & -s_{7} c_{8} \\
0 & 0 & 0 & -1 & 0 & s_{8} \\
-\frac{1}{d_{7}^{\prime}} & 0 & 0 & 0 & -s_{7} & c_{7} c_{8}
\end{array}\right),
$$

with $\mathbf{v}_{9}$ resp. $\omega_{9}$ the translational and rotational velocity of frame $\{\mathbf{9}\}$ and $\left(\dot{x}_{6}, \dot{y}_{6}, \dot{z}_{6}\right)$ the translational velocities of frame $\{\mathbf{W}\}$. The determinant of ${ }_{9}^{6} \mathbf{J}_{v}$ yields:

$$
\left|{ }_{9}^{6} \mathbf{J}_{v}\right|=-\frac{\left(d_{7, a}-d_{7}\right)^{2} c_{8}}{d_{7, a}^{2}},
$$

and a singular configuration can be stated when

$$
c_{8}=0 \text {, }
$$

with the axes $z_{7}$ and $z_{9}$ aligned. The singular configuration that occurs if

$$
d_{7, a}=d_{7}
$$

corresponds to a configuration where the fulcrum point is coincident with the origin of $\{\mathbf{W}\}$. In this case, translations of the frame $\{\mathbf{W}\}$ are partly restricted by the constraint of the fulcrum point, and an altered Jacobian matrix (a matrix that takes into consideration the rotation of 


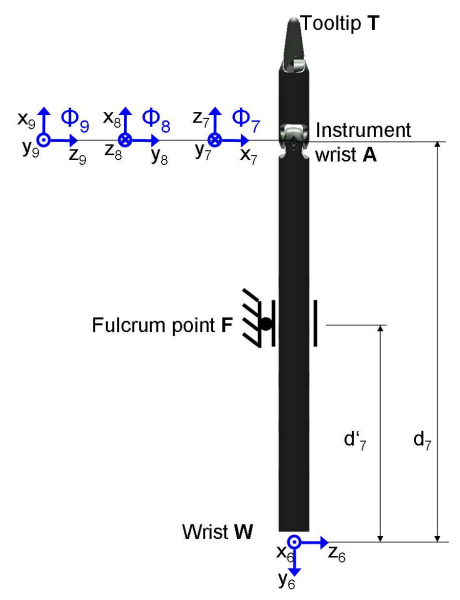

Figure 3. Kinematic description of the articulated instrument in MIS. The length $d_{7}^{\prime}$ signifies the distance between the wrist frame $\{\mathbf{W}\}$ and the fulcrum point.

frame $\{\mathbf{W}\}$ rather than its translation) would have to be considered. Since, due to the design of the considered robot, the wrist joint cannot be coincident with the fulcrum point this case is not further analyzed.

\section{Conclusion}

In this paper the analytical solution for the determination of all singularities of the DLR medical robot with attached articulated instrument is given. The use of the formula of Cauchy-Binet simplifies the equations considerably and is suggested for the calculation of the singularities of similar redundant kinematic structures. Particularly, the singular configurations of both the DLR light weight robots II and III (7-DoF robots) can be easily determined. As for the DLR medical robot, all singularities except for $e_{1}\left(\phi_{4}=\pi k\right)$ and $e_{5}\left(\phi_{5}=\frac{\pi}{2}+\pi k \wedge \phi_{6}=\pi k\right)$ are outside of the joint limits.

\section{Appendix}

The relevant determinants yield:

$$
\begin{gathered}
\left|\mathbf{J}_{22}\right|=-s_{6}, \quad\left|\mathbf{J}_{11}^{1}\right|=-a_{3} d_{5} c_{3} s_{4}\left(d_{5} c_{4}+a_{3}\right), \\
\left|\mathbf{J}_{11}^{2}\right|=a_{3} d_{5} c_{2} s_{3} s_{4}\left(d_{5} c_{4}+a_{3}\right), \quad\left|\mathbf{J}_{11}^{3}\right|=a_{3} d_{5} s_{4}\left(s_{2} c_{3}\left(a_{3}+d_{5} c_{4}\right)+d_{5} c_{2} s_{4}\right),
\end{gathered}
$$




$$
\begin{gathered}
\left|\mathbf{J}_{11}^{4}\right|=0, \quad\left|\left(\begin{array}{cc}
\mathbf{J}_{11} & \mathbf{0} \\
\mathbf{J}_{21} & \mathbf{J}_{22}^{1}
\end{array}\right)\right|=-a_{3} d_{5} c_{2} s_{4}\left(d_{5} s_{6}+a_{3} s_{4} c_{5} c_{6}+a_{3} c_{4} s_{6}\right), \\
\left|\left(\begin{array}{cc}
\mathbf{J}_{11} & \mathbf{0} \\
\mathbf{J}_{21} & \mathbf{J}_{22}^{2}
\end{array}\right)\right|=a_{3}^{2} d_{5} c_{2} s_{4}^{2} s_{5} s_{6}, \quad\left|\left(\begin{array}{cc}
\mathbf{J}_{11} & \mathbf{0} \\
\mathbf{J}_{21} & \mathbf{J}_{22}^{3}
\end{array}\right)\right|=a_{3}^{2} d_{5} c_{2} s_{4} c_{5} ;
\end{gathered}
$$

The determinants equal zero for the following joint angles:

$$
\begin{aligned}
& \left|\mathbf{J}_{11}^{1}\right|=0: \quad \phi_{3}=\frac{\pi}{2}+\pi k \quad \vee \quad \phi_{4}=\pi k \quad \vee \quad \phi_{4}= \pm \arccos \left(-\frac{a_{3}}{d_{5}}\right)+2 \pi k ; \\
& \left|\mathbf{J}_{11}^{2}\right|=0: \quad \phi_{2}=\frac{\pi}{2}+\pi k \vee \phi_{3}=\pi k \vee \phi_{4}=\pi k \vee \phi_{4}= \pm \arccos \left(-\frac{a_{3}}{d_{5}}\right)+2 \pi k \\
& \left|\mathbf{J}_{11}^{3}\right|=0: \quad \phi_{4}=\pi k \quad \vee \quad \phi_{2}= \pm \arctan \left(\frac{s_{4} d_{5}}{c_{3}\left(d_{5} c_{4}+a_{3}\right)}\right)+\pi k, \\
& \left|\mathbf{J}_{11}^{4}\right|=0 \forall \phi_{i} ; \\
& \left|\left(\begin{array}{cc}
\mathbf{J}_{11} & \mathbf{0} \\
\mathbf{J}_{21} & \mathbf{J}_{22}^{1}
\end{array}\right)\right|_{s_{6}=0}=0: \phi_{2}=\frac{\pi}{2}+\pi k \quad \vee \quad \phi_{4}=\pi k \\
& \left|\left(\begin{array}{cc}
\mathbf{J}_{11} & \mathbf{0} \\
\mathbf{J}_{21} & \mathbf{J}_{22}^{2}
\end{array}\right)\right|_{s_{6}=0}=0, \forall \phi_{i} \\
& \left|\left(\begin{array}{cc}
\mathbf{J}_{11} & \mathbf{0} \\
\mathbf{J}_{21} & \mathbf{J}_{22}^{3}
\end{array}\right)\right|_{s_{6}=0}=0: \phi_{2}=\frac{\pi}{2}+\pi k \quad \vee \quad \phi_{4}=\pi k \quad \vee \quad \frac{\pi}{2}+\pi k ;
\end{aligned}
$$

\section{References}

Louaï Adhami. An Architecture for Computer Integrated Mini-Invasive Robotic Surgery: Focus on Optimal Planning. Ph.D. Thesis, Ecole des Mines de Paris, Paris, 2002.

Felix R. Gantmacher. The Theory of Matrices. American Mathematical Society, 1959.

Rainer Konietschke, Tobias Ortmaier, Holger Weiss, Gerd Hirzinger, and Robert Engelke. Manipulability and Accuracy Measures for a Medical Robot in Minimally Invasive Surgery. In Advances in Robot Kinematics, Genua, Italy, 2004.

Rainer Konietschke, Holger Weiss, Tobias Ortmaier, and Gerd Hirzinger. A Preoperative Planning Procedure for Robotically Assisted Minimally Invasive Interventions. In 3. Jahrestagung der Deutschen Gesellschaft für Computer- und Roboterassistierte Chirurgie (CURAC), München, Germany, 8.-9. Dezember 2004.

Tsuneo Yoshikawa. Foundations of Robotics: Analysis and Control. The MIT Press, 1990. 